The Astrophysical Journal, 450:350-363, 1995 September 1 .

(C) 1995. The American Astronomical Society. All rights reserved. Printed in U.S.A.

\title{
WHOLE EARTH TELESCOPE OBSERVATIONS AND SEISMOLOGICAL ANALYSIS OF THE PRE-WHITE DWARF PG $2131+066$
}

\author{
S. D. Kawaler ${ }^{1}$ M. S. O’Brien, ${ }^{1}$ J. C. Clemens, ${ }^{1,2,3}$ R. E. Nather, ${ }^{3}$ D. E. Winget, ${ }^{3}$ T. K. Watson, ${ }^{3}$ \\ K. Yanagida, ${ }^{3,4}$ J. S. Dixson, ${ }^{3}$ P. A. Bradley, ${ }^{3,5}$ M. A. Wood, ${ }^{6,7}$ D. J. Sullivan, ${ }^{8}$ S. J. Kleinman, ${ }^{3,9}$ \\ E. Meištas, ${ }^{10,11}$ E. M. Leibowitz, ${ }^{12}$ P. Moskalik, ${ }^{13}$ S. Zola, ${ }^{14,15}$ G. PAJdosz, ${ }^{14}$ J. KrzesińsKi, ${ }^{14}$ \\ J.-E. Solheim, ${ }^{11,16}$ A. Bruvold, ${ }^{16}$ D. O'Donoghue, ${ }^{17}$ M. Katz, ${ }^{17}$ G. Vauclair, ${ }^{18}$ \\ N. Dolez, ${ }^{18}$ M. Chevreton, ${ }^{19}$ M. A. Barstow, ${ }^{20}$ A. KanaAn, ${ }^{3,21}$ S. O. KePler, ${ }^{22}$ \\ O. Giovannini, ${ }^{22,23}$ J. L. Provencal, ${ }^{3,24,25}$ and C. J. Hansen ${ }^{26}$ \\ Received 1994 December 1; accepted 1995 March 14
}

\begin{abstract}
As transition objects between the asymptotic giant branch (AGB) and cooling white dwarfs, the PG 1159 stars are key objects for understanding the late stages of stellar evolution. The pulsations exhibited by many members of the PG 1159 spectral class provide a mechanism for ferreting out this information. We present an analysis of the pulsation spectrum of the naked PG 1159 star PG $2131+066$, which we completely resolve using data from the Whole Earth Telescope (WET). The pulsation spectrum shows patterns that are expected from pulsation theory; comparison of these patterns with theoretical models indicate that PG 2131 has a mass of $0.61 \pm 0.02 M_{\odot}$, a luminosity of $\log \left(L / L_{\odot}\right)=1.0 \pm 0.2$, and it lies at a distance of $470_{-130}^{+180}$ pc. It rotates with a period of $5.1 \mathrm{hr}$ and shows evidence of a discontinuity in composition approximately $6 \times 10^{-3} M_{*}$ below the surface. Certain modes show complex structure in the power spectrum, while others show measurable frequency changes in 8 years. Fine structure in the prograde $(m=-1)$ modes suggests the presence of nonlinear effects.
\end{abstract}

Subject headings: stars: fundamental parameters - stars: individual (PG $2131+066)$ - stars: oscillations white dwarfs

\section{INTRODUCTION}

The star PG $2131+066$ (a.k.a. IR Peg, hereafter PG 2131) is a member of the PG 1159 spectral class of hot and luminous pre-white dwarf stars. These stars are the direct descendants of the central stars of planetary nebulae, and the precursors to more ordinary white dwarfs. As such, they are the transition objects between stars with active nuclear burning (AGB stars) and stars that are destined to simply cool and fade (white dwarf stars). Probing their interior structure provides direct constraints on the transition from AGB to white dwarf and on the properties of both classes of stars.

${ }^{1}$ Department of Physics and Astronomy, Iowa State University, Ames, IA 50011; sdk@iastate.edu.

${ }^{2}$ Hubble Fellow.

${ }^{3}$ Department of Astronomy, University of Texas, Austin, TX 78712.

4 5-35-11 Hongōdai, Sakae-ku Yokohama 247, Japan.

5 X-2, MS B-220, Los Alamos National Laboratory, Los Alamos, NM 87545.

${ }^{6}$ Department of Physics and Space Science, Florida Institute of Technology, 150 W. University Boulevard, Melbourne, FL 32901.

7 Guest Observer, Institute for Astronomy, Honolulu, HI.

${ }^{8}$ Department of Physics, Victoria University, Box 600, Wellington, New Zealand.

${ }^{9}$ Guest Observer, Mount Stromlo and Siding Spring Observatory, N.S.W., Australia.

${ }^{10}$ Institute of Theoretical Physics and Astronomy, Goštauto 12, Vilnius 2600, Lithuania.

${ }^{11}$ Guest Observer, Maidanak Observatory, Uzbekistan.

12 Department of Physics and Astronomy, University of Tel Aviv, Ramat Aviv, Tel Aviv 69978, Israel.

${ }^{13}$ Copernicus Astronomical Center, ul. Bartycka 18, 00-716 Warsaw, Poland.

${ }^{14}$ Mount Suhora Observatory, Kraków Pedagogical University, ul. Podchorążych 2, 30-084 Kraków, Poland.
A new and powerful tool for probing the depths of these stars is temporal spectroscopy. This is the principal observational tool in the area of asteroseismology, and can be applied to those objects that show periodic photometric variability. In less esoteric terms, those stars that are pulsators reveal their internal structure through the periods of their photometric variations. The prototype of the pulsating hot white dwarfs, PG 1159-035 (or GW Vir), has yielded a wealth of information about what one of these stars look like on the inside, through analysis of its light curve as obtained by the Whole Earth Telescope (WET; Nather et al. 1990) in 1989. The report of these observations and their analysis (Winget et al. 1991, hereafter "WWET") shows what such stars can teach us.

\footnotetext{
15 Astronomical Observatory, Jagiellonian Univ., ul. Orla 171, 30-244 Kraków, Poland.

${ }^{16}$ Institutt for Matematiske Realfag, Universitet i Tromso, 9000 Tromso, Norway.

${ }^{17}$ Department of Astronomy, University of Cape Town, Rondebosch 7700, Cape Province, South Africa.

${ }^{18}$ Observatoire Midi-Pyrenees, 14 Avenue E. Belin, 31400 Toulouse, France.

${ }^{19}$ Observatoire de Paris-Meudon, F-92195 Meudon, Principal Cedex, France.

${ }^{20}$ Physics Department, University of Leicester, Leicester LE1 7RH, UK.

${ }^{21}$ Guest Observer, Isaac Newton Telescope, Roque de los Muchachos, La Palma, Canaries.

${ }^{22}$ Instituto de Fisica, Universidade Federal do Rio Grande do Sul, 91501970 Porto Alegre-RS, Brazil.

${ }^{23}$ Visiting Astronomer, Laboratorio Nacional de Astrofisica, CNPq, Brazil.

${ }^{24}$ University of Delaware, Physics and Astronomy Department, Sharp Laboratory, Newark, DE 19716.

${ }^{25}$ Guest Observer, CTIO (NOAO/AURA), Chile.

26 J.I.L.A., University of Colorado, Box 440, Boulder, CO 80309.
} 
However, PG 1159 is wonderful in that it shows all that we expect to see (and more) in these stars. An unbroken sequence of dozens of periodicities match very nicely with the expectations from stellar evolution and pulsation theory. The other GW Vir stars are less obliging. Until WET analysis, PG 1707, PG 2131, PG 0122, and RX J2117 showed only a few modes, allowing little in the way of analysis beyond superficial comparison with PG 1159.

PG 2131 itself was little observed (photometrically) following its discovery as a variable star by Bond et al. (1984). In part this is because it is relatively faint $(V=16.6, B-V=-0.34$; Bond et al. 1984). Though observed several times at McDonald Observatory in the mid-1980s, the observations were not published. Bond et al. (1984) show PG 2131 had periods of about 414 and $386 \mathrm{~s}$, along with some other periodicities. The McDonald data indicated at least six additional periodicities, but the limitations of single-site observing rendered positive period determinations impossible. The Kiel group has analyzed the spectrum of PG 2131, and report an effective temperature of about $80,000 \pm 10,000$ Kelvin, and $\log$ $g=7.5 \pm 0.5$ (Dreizler et al. 1995). For comparison, PG 1159 has an effective temperature of $140,000 \mathrm{~K}$ and $\log g=7.0 \pm 0.5$ from spectroscopic studies (Werner, Heber, \& Hunger 1991, hereafter WHH), while Kawaler \& Bradley (1994) found $T_{\text {eff }} \sim$ $136,000 \mathrm{~K}$ and $\log g=7.4 \pm 0.1$. Dreizler et al. (1995) indicate that PG 2131 might be more helium rich than PG 1159.

One thing that sets PG 2131 apart from the other PG 1159 stars is that its optical spectrum shows an apparent red excess, which has been attributed to a nearby cool companion (Wesemael, Green, \& Liebert 1985). Wesemael et al. used the excess to estimate the spectral type of the companion as $\mathrm{K} 7 \pm 1$. The binary nature of PG 2131 was dramatically confirmed by H. E. Bond (1994, private communication) with an HST image which shows a very red companion star 0 ".3 away from PG
2131 itself. The companion is as red as PG 2131 is blue; it outshines PG 2131 in the $I$ band, while it is barely visible in the $V$ exposure. At a distance of $470 \mathrm{pc}$, the angular separation corresponds to a minimum orbital radius of approximately 140 $\mathrm{AU}$; thus, this is (for the purposes of asteroseismology) a wide binary. As a result of our use of blue-sensitive phototubes, the companion is only seen in our photometry as a slight increase in sky counts, if at all.

WWET and Kawaler \& Bradley (1994), through their analyses of PG 1159, confirmed that nonradial pulsation theory is in basic agreement with at least one real star. Armed with this confidence, we can attempt to understand a less obliging subject; PG 2131 is a good candidate for an in-depth analysis with the WET. As we show below, it is not as rich a pulsator as PG 1159. However, we still obtain a precise mass determination, place limits on the surface compositional stratification, measure its rotation rate, and place limits on the global magnetic field strength. The results of this analysis provide a second data point in the determination of the class properties of the PG 1159 stars, and put us on track for a complete description of these stars. Papers in preparation by the WET collaboration will describe observations of other members of this class; eventually we will have enough pieces to solve the puzzle of the late stages of evolution of stars like our Sun.

\section{OBSERVATIONS}

To some extent, the 1992 September WET run that had PG 2131 as its primary target was a "typical" WET run. Time series photometric data were obtained at several observatories around the globe; the observatories involved and the resulting runs are listed in the journal of observations in Table 1. Data were obtained and reduced following the procedures described

TABLE 1

Table Of Observations of PG 2131 FOR the WET Coverage IN 1992

\begin{tabular}{|c|c|c|c|c|}
\hline Telescope & Run Name & $\begin{array}{l}\text { Date } \\
\text { (UT) }\end{array}$ & $\begin{array}{l}\text { Start Time } \\
\text { (UT) }\end{array}$ & $\begin{array}{l}\text { Run Length } \\
\text { (h:m:s) }\end{array}$ \\
\hline Mount John & se2192 & 1992 Sep 21 & $08: 10: 35$ & $5: 29: 25$ \\
\hline La Palma INT. & int -0008 & 1992 Sep 21 & $23: 19: 44$ & $3: 32: 40$ \\
\hline CTIO $1.5 \mathrm{~m} \ldots \ldots \ldots$ & jlp-0110 & 1992 Sep 22 & $01: 26: 00$ & $4: 02: 25$ \\
\hline McDonald 82 inch & pab-0143 & 1992 Sep 22 & $06: 55: 20$ & $1: 30: 30$ \\
\hline SAAO $0.75 \mathrm{~m} \ldots \ldots$ & s5526 & 1992 Sep 22 & $19: 26: 31$ & $1: 30: 50$ \\
\hline La Palma INT.. & int-0011, 12 & 1992 Sep 22 & $21: 20: 47$ & $4: 45: 53$ \\
\hline CTIO $1.5 \mathrm{~m} \ldots .$. & jlp-0113 & 1992 Sep 23 & $00: 02: 00$ & $6: 07: 35$ \\
\hline LNA $1.6 \mathrm{~m} \ldots$ & ro020 & 1992 Sep 23 & $23: 33: 00$ & $1: 11: 30$ \\
\hline CTIO $1.5 \mathrm{~m}$. & jlp-0115 & 1992 Sep 24 & $00: 06: 25$ & $4: 51: 35$ \\
\hline Mauna Kea 24 inch...... & maw-0102 & 1992 Sep 24 & $06: 18: 00$ & $6: 59: 05$ \\
\hline SAAO $0.75 \mathrm{~m} \ldots \ldots \ldots \ldots$ & s5527 & 1992 Sep 24 & $21: 18: 26$ & $0: 55: 20$ \\
\hline OHP $1.93 \mathrm{~m} \ldots \ldots \ldots \ldots$ & gv-0231 & 1992 Sep 24 & $22: 03: 30$ & $3: 28: 00$ \\
\hline CTIO $1.5 \mathrm{~m} \ldots$ & jlp-0118 & 1992 Sep 24 & $23: 55: 00$ & $6: 18: 45$ \\
\hline McDonald 82 inch ....... & pab-0153 & 1992 Sep 25 & 06:06:05 & $2: 51: 30$ \\
\hline Mauna Kea 24 inch...... & maw-0104 & 1992 Sep 25 & $08: 44: 20$ & $4: 23: 00$ \\
\hline OHP $1.93 \mathrm{~m} \ldots \ldots \ldots \ldots$ & gv-0233 & 1992 Sep 25 & $19: 09: 30$ & $6: 08: 20$ \\
\hline CTIO $1.5 \mathrm{~m} \ldots$ & jlp-0120, 21 & 1992 Sep 25 & $23: 56: 10$ & $6: 21: 20$ \\
\hline McDonald 82 inch ...... & pab-0157 & 1992 Sep 26 & $05: 59: 40$ & $3: 10: 00$ \\
\hline SSO 40 inch $\ldots \ldots \ldots \ldots \ldots$ & sjk-0198 & 1992 Sep 26 & $11: 30: 50$ & $2: 58: 15$ \\
\hline CTIO $1.5 \mathrm{~m} \ldots \ldots \ldots \ldots \ldots$ & jlp-0123 & 1992 Sep 27 & $00: 13: 25$ & $4: 55: 40$ \\
\hline Mauna Kea 24 inch...... & maw-0105 & 1992 Sep 27 & $05: 15: 10$ & $1: 30: 25$ \\
\hline SSO 40 inch $\ldots \ldots \ldots \ldots . .$. & sjk-0201 & 1992 Sep 27 & $09: 32: 30$ & $6: 25: 45$ \\
\hline SAAO $0.75 \mathrm{~m} \ldots \ldots \ldots \ldots$ & s5529 & 1992 Sep 27 & $19: 44: 38$ & $2: 33: 25$ \\
\hline CTIO $1.5 \mathrm{~m} \ldots \ldots \ldots \ldots \ldots$ & jlp-0126 & $1992 \operatorname{Sep} 28$ & $02: 12: 45$ & $3: 50: 30$ \\
\hline SAAO $0.75 \mathrm{~m} \ldots \ldots \ldots \ldots$ & s5531 & 1992 Sep 29 & $18: 27: 25$ & $1: 57: 00$ \\
\hline AAO $0.75 \mathrm{~m} \ldots \ldots$ & s5534 & 1992 Sep 30 & $18: 03: 24$ & $4: 43: 20$ \\
\hline
\end{tabular}


by Nather et al. (1990) and WWET. A total of 70,552 5 second integrations were obtained at all observatories over a 9.6 day period, resulting in an overall duty cycle of $42 \%$. During the most intensive portion of the run, the first 7 days, the duty cycle was $55 \%$. Times within all runs used the common zero time of 2448886.84472 BJED, which corresponds to the first integration of the run. In final form, each individual run is represented by the time of each integration and the fractional departure of the count rate from the mean. In our analysis, the data were summed to produce $40 \mathrm{~s}$ integrations. This greatly eased the computational burden of our analysis, but sacrificed sensitivity to periods shorter than about $100 \mathrm{~s}$ (though no signals were seen at periods shorter than $335 \mathrm{~s}$ ). Doing this also led to a slightly degraded frequency precision (increasing the least-squares uncertainty in frequency from 0.2 to $0.4 \mu \mathrm{Hz}$ ).

The data are shown in Figure 1 in $24 \mathrm{hr}$ strips from the beginning of the run. One sees immediately from this figure that there remains a gap in the coverage that corresponds to a gap in the global network. This gap (at about 32,000 s) would have been filled nicely by the site at Mount Maidanak Observatory in Uzbekistan. While two of us (J. E. S. and E. M.) did obtain some data at Mount Maidanak, weather conditions and technical problems at this remote site were less than ideal, so the data were too noisy for inclusion in the final data set. As a result, the window for this run has higher 1 cycle day $^{-1}$ sidelobes than previous WET observations. The window function for this run, obtained by sampling a single-frequency sine wave of fixed amplitude at the observed sample times, is shown in Figure 2. We remain optimistic that the Mount Maidanak observing site will be extremely useful in future WET campaigns.

We computed the Fourier transform of the reduced data set using a brute-force discrete Fourier transform, and squaring the amplitude of the complex transform to produce the power spectrum. This then gives the total modulation power $(\mathrm{mp})$ as a function of frequency. The square root of the modulation power gives the modulation amplitude. In many cases, we find that plots of modulation amplitude (ma; a.k.a. amplitude spectra) are more useful than power spectra, as the amplitude spectra de-emphasize strong peaks. This representation of pulsation power and amplitude in units of $\mathrm{mp}$ and ma is discussed in Winget et al. (1994). The power spectrum is shown in Figure 3 for the entire frequency range of interest. Note that not all horizontal panels have the same scale in power. The band of largest power is between 1900 and $3000 \mu \mathrm{Hz}$; Figure 4 expands this region, and shows both the power spectrum and the amplitude spectrum for further comparison. The three largest amplitude modes appear at frequencies of 2575.5, 2447.2, and 2629.5 $\mu \mathrm{Hz}$, with five additional peaks present that do not correspond to diurnal aliases of other peaks.

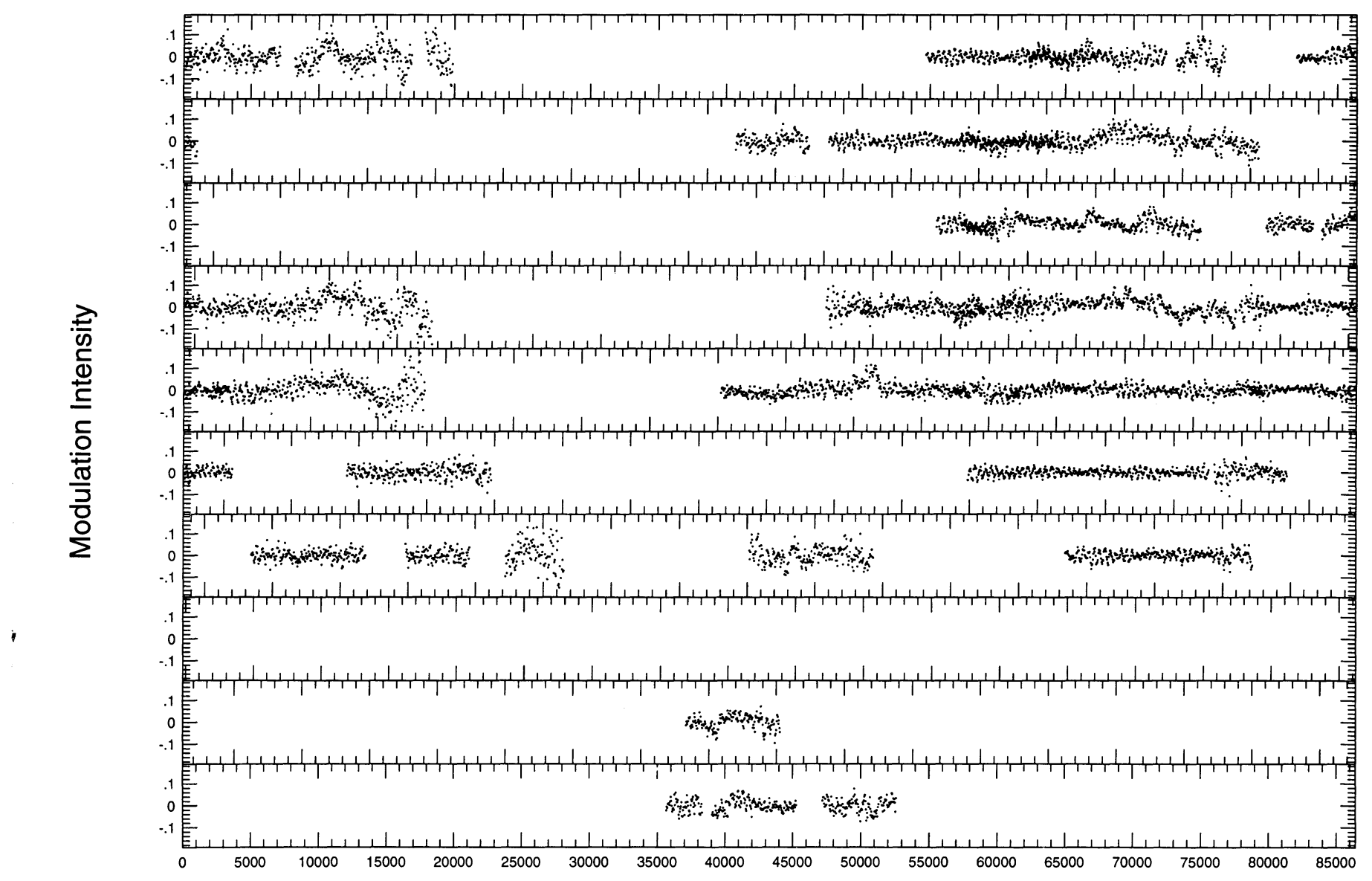

Time [s]

FIG. 1.-Combined light curve of PG 2131. Each horizontal panel represents $24 \mathrm{hr}$ of data. 


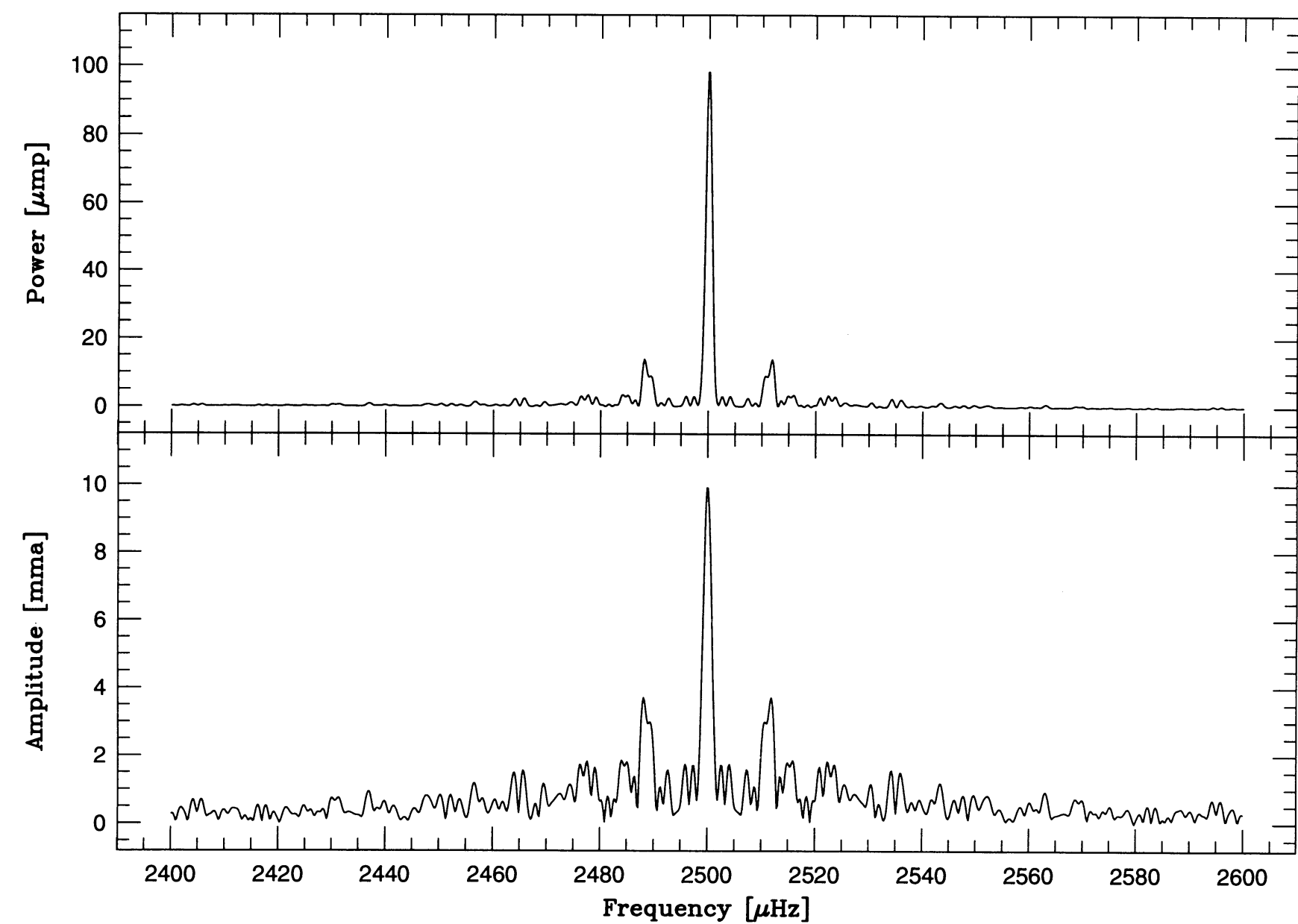

FIG. 2.-Spectral window of the WET run. The top panel shows the power spectrum of the window function, while the bottom panel shows the amplitude spectrum. Aside from the 1 cycle day $^{-1}$ sidelobe, this window was sufficiently clean to allow unambiguous identification of many peaks in the spectrum.

In practice, the 1 cycle day $^{-1}$ alias, and the resulting window pattern shown in Figure 2, results in unattractive but unconfusing false peaks in the power spectra. These sidelobes are low enough in amplitude that they do not interfere with identification of large-amplitude peaks. However, the sidelobes do interfere with identifying low-amplitude peaks, and in precise measurement of amplitudes and frequencies for closely spaced peaks. To address these problems, we used a linear leastsquares fitting procedure to determine the precise frequencies, amplitudes, and phases of many peaks simultaneously. This procedure allows accurate determinations of the amplitudes of the modes included in the fit that are hidden but not influenced by the sampling pattern.

We used the amplitude spectrum of the entire data set to provide initial guesses for nine of the highest peaks, with frequencies greater than $1000 \mu \mathrm{Hz}$, for use in the initial leastsquares fit. In selecting these peaks, we excluded those that were clearly related to cycle day ${ }^{-1}$ aliases of other peaks. The least-squares identifications match in frequency with peaks in the amplitude spectrum within the errors. However, the amplitudes are different for the reason stated above; the leastsquares amplitudes and frequencies are more accurate. We then prewhitened the original data by the nine frequencies found above to try and recover lower amplitude peaks. To prewhiten, we computed an artificial signal by adding together sine curves with the amplitudes, frequencies, and times of maximum found by the least-squares analysis and sampled this signal at the same times as the data. By subtracting this signal from the original data, we obtained a data set that contains all periodicities except the nine large-amplitude ones. Power and amplitude spectra of the prewhitened data set are shown in Figure 5. Prewhitening reveals several additional periodicities in the data set. The eight largest peaks in the interval between 1900 and $3000 \mu \mathrm{Hz}$ were included in the list that makes up Table 2. Two of the three remaining peaks (at 2953 and 2929 $\mu \mathrm{Hz}$ ) were the largest in a $100 \mu \mathrm{Hz}$ region of the prewhitened power spectrum, except for a peak at the $1 \mathrm{cycle}^{\mathrm{day}}{ }^{-1}$ alias of the 2953 peak. The final peak, at $2221 \mu \mathrm{Hz}$, was also the highest peak in the surrounding $100 \mu \mathrm{Hz}$ region not already considered. A final least-squares fit to the data including all the identified peaks gave us the parameters listed in Table 2.

We resolved 20 independent periodicities in the combined WET run for PG 2131. As indicated in Table 2, there is one peak that is almost resolved into two (at $2953 \mu \mathrm{Hz}$ ); it shows broadened line shapes in the spectra. The $2503 \mu \mathrm{Hz}$ peak has three components in the combined data set; we could resolve two of them, but the lower frequency component was not resolved. Two additional closely spaced pairs are present in Table 2 that were resolved in this analysis. We will show later that all of these close peaks share other distinguishing features. 
TABLE 2

Periodicities of PG $2131+066$ From WET Data.

\begin{tabular}{|c|c|c|c|c|c|c|}
\hline $\begin{array}{l}\text { Frequency } \\
\qquad(\mu \mathrm{Hz})\end{array}$ & $\begin{array}{l}\text { Period } \\
\text { (s) }\end{array}$ & $\begin{array}{l}\text { Amplitude } \\
\text { (mma) }\end{array}$ & $\underset{(\mathrm{max}}{T^{\mathrm{a}}}$ & $m$ & $n$ & Comments $^{b}$ \\
\hline $\begin{array}{l}2953.25 \pm 0.25 \ldots \ldots \\
2928.66 \pm 0.31 \ldots \ldots \\
2900.98 \pm 0.17 \ldots \ldots\end{array}$ & $\begin{array}{l}338.610 \pm 0.029 \\
341.453 \pm 0.036 \\
344.711 \pm 0.020\end{array}$ & $\begin{array}{l}1.42 \pm 0.48 \\
1.27 \pm 0.48 \\
2.23 \pm 0.48\end{array}$ & $\begin{array}{r}68 \pm 37 \\
178 \pm 41 \\
264 \pm 23\end{array}$ & $\begin{array}{r}-1 \\
0 \\
1\end{array}$ & 13 & $\begin{array}{c}+1.2 \mu \mathrm{Hz} \text { split } \\
\text { LSQ }\end{array}$ \\
\hline $\begin{array}{l}2629.44 \pm 0.06 \ldots \ldots \\
2602.34 \pm 0.14 \ldots \ldots \\
2575.55 \pm 0.04 \ldots \ldots\end{array}$ & $\begin{array}{l}380.309 \pm 0.009 \\
384.269 \pm 0.021 \\
388.266 \pm 0.006\end{array}$ & $\begin{array}{r}6.34 \pm 0.48 \\
2.69 \pm 0.48 \\
11.01 \pm 0.48\end{array}$ & $\begin{aligned} 228 & \pm 9 \\
26 & \pm 22 \\
87 & \pm 5\end{aligned}$ & $\begin{array}{c}-1 \\
0: \\
1\end{array}$ & 15 & $\begin{array}{l}\text { LSQ } \\
\text { LSQ }\end{array}$ \\
\hline $\begin{array}{l}2503.50 \pm 0.15 \ldots \ldots \\
2500.63 \pm 0.19 \ldots \ldots \\
2475.67 \pm 0.25 \ldots \ldots \\
2447.17 \pm 0.04 \ldots \ldots\end{array}$ & $\begin{array}{l}399.441 \pm 0.024 \\
399.899 \pm 0.027 \\
403.930 \pm 0.041 \\
408.635 \pm 0.007\end{array}$ & $\begin{array}{l}2.83 \pm 0.49 \\
2.24 \pm 0.49 \\
1.62 \pm 0.49 \\
9.73 \pm 0.48\end{array}$ & $\begin{array}{l}345 \pm 22 \\
378 \pm 29 \\
396 \pm 39 \\
404 \pm 6\end{array}$ & $\begin{array}{c}-1: \\
0: \\
1\end{array}$ & 16 & LSQ \\
\hline $\begin{array}{l}2373.56 \pm 0.13 \ldots \ldots \\
2372.18 \pm 0.14 \ldots \ldots \\
2345.45 \pm 0.21 \ldots \ldots \\
2318.85 \pm 0.10 \ldots \ldots\end{array}$ & $\begin{array}{l}421.307 \pm 0.023 \\
421.553 \pm 0.024 \\
426.358 \pm 0.038 \\
431.248 \pm 0.019\end{array}$ & $\begin{array}{l}3.81 \pm 0.50 \\
3.39 \pm 0.50 \\
1.90 \pm 0.48 \\
4.05 \pm 0.48\end{array}$ & $\begin{array}{l}382 \pm 22 \\
298 \pm 25 \\
189 \pm 34 \\
110 \pm 16\end{array}$ & $\begin{array}{c}-1: \\
0: \\
1\end{array}$ & 17 & $\begin{array}{l}\text { LSQ } \\
\text { LSQ } \\
\text { LSQ }\end{array}$ \\
\hline $\begin{array}{l}2249.24 \pm 0.16 \ldots \ldots \\
2247.56 \pm 0.26 \ldots \ldots \\
2220.86 \pm 0.28 \ldots \ldots \\
2192.31 \pm 0.18 \ldots \ldots\end{array}$ & $\begin{array}{l}444.595 \pm 0.032 \\
444.927 \pm 0.051 \\
450.276 \pm 0.057 \\
456.139 \pm 0.035\end{array}$ & $\begin{array}{l}2.68 \pm 0.48 \\
1.71 \pm 0.49 \\
1.42 \pm 0.48 \\
2.21 \pm 0.48\end{array}$ & $\begin{aligned} 389 & \pm 29 \\
39 & \pm 46 \\
418 & \pm 48 \\
392 & \pm 31\end{aligned}$ & $\begin{array}{c}-1: \\
0 \\
1\end{array}$ & 18 & LSQ \\
\hline $2162.68 \pm 0.11 \ldots \ldots$ & $462.390 \pm 0.024$ & $3.70 \pm 0.48$ & $378 \pm 19$ & -1 & 19 & LSQ \\
\hline $1968.87 \pm 0.19 \ldots \ldots$ & $507.906 \pm 0.049$ & $2.02 \pm 0.48$ & $386 \pm 38$ & $-1:$ & 21 & \\
\hline
\end{tabular}

Time of first maximum after BJED 2448886.84472

b "LSQ" labels the nine peaks in first least-squares fit to original data.

\section{RESULTS}

\subsection{Frequency Splitting}

One of the observed properties of nonradial oscillations is that equal frequency spacings are often seen when "bands" of power are resolved. This is the result of departures from pure spherical symmetry in the star breaking a degeneracy in the pulsation frequencies. In the absence of such departures (that can be caused by rotation, magnetic fields, or some combination), the frequencies of nonradial modes with a given degree $\ell$ and radial order $n$ are identical. ${ }^{27}$ Rotation, for example, lifts this degeneracy and splits the degenerate modes into $2 \ell+1$ components (if all values of the azimuthal index $m$ are present) as follows:

$$
v_{n l m}=v_{n l 0}-m \frac{1}{P_{\mathrm{rot}}}\left(1-C_{n l}\right)
$$

where for white dwarf stars, to within a few percent,

$$
C_{n l}=\frac{1}{\ell(\ell+1)}
$$

(Brickhill 1975; WWET). Thus, we expect to see triplets split by one-half of the rotation frequency for $\ell=1$ modes. Since the dominant modes in PG 1159 are all $\ell=1$ modes, we expect to see $\ell=1$ modes in PG 2131 as well. We follow the convention that the lower frequency component of a triplet is an

${ }^{27}$ We use $n$ as the mode order to maintain consistency with Kawaler \& Bradley (1994), and with the general usage in nonradial stellar pulsation theory (i.e., Unno et al. 1989; Cox 1980). The value of $n$ can differ from $k$ (the number

- of nodes in the radial component of the displacement eigenfunction) in centrally concentrated stars (such as red giants); however, $n$ and $k$ are almost always equal in white dwarf models. $m=+1$ mode, and the higher frequency component is an $m=-1$ mode. This choice differs from that made by Winget et al. $(1991,1994)$ in previous WET reports; here we use this convention to maintain consistency with the standard phase convention for spherical harmonics used in most areas of physics (see, e.g., Jackson 1975, $\S 3.5$, and references therein) and in the standard texts of stellar seismology (i.e., Unno et al. 1989; Cox 1980).

With the hope and expectation that we would see the signature of rotational splitting, we searched for equal frequency spacings between the observed peaks in the initial (nonprewhitened) power spectrum of PG 2131. We found five pairs of peaks, all split by approximately $54 \mu \mathrm{Hz}$. Since rotational splitting gives $2 \ell+1$ peaks (an odd number), and since triplets $(\ell=1)$ are seen in other pulsating white dwarf stars (i.e., Winget et al. 1991, 1994), we looked for a central component between the large peaks. Three of these pairs showed hints of a central peak almost exactly in the middle, suggesting that they were triplets with spacings of $27 \mu \mathrm{Hz}$. Upon prewhitening (see the previous section), we found that all five pairs show significant peaks within $1.5 \mu \mathrm{Hz}$ of the midpoint in frequency. These five apparent triplets show a mean splitting of $27.4 \pm 1.2 \mu \mathrm{Hz}$

Figure 6 shows the power spectrum surrounding the proposed triplets seen in PG 2131 with the low-frequency components aligned. The segments of power spectrum shown in Figure 6 are (necessarily) from the original power spectrum; window effects and the use of power as the ordinate frequently hide the apparent significance of the small-amplitude peak we identify as the $m=0$ component. We display the averaged spectra of the five regions of power in the bottom panel of Figure 7. The top panel shows the averaged power spectra when they are plotted relative to the central peak. In construc- 


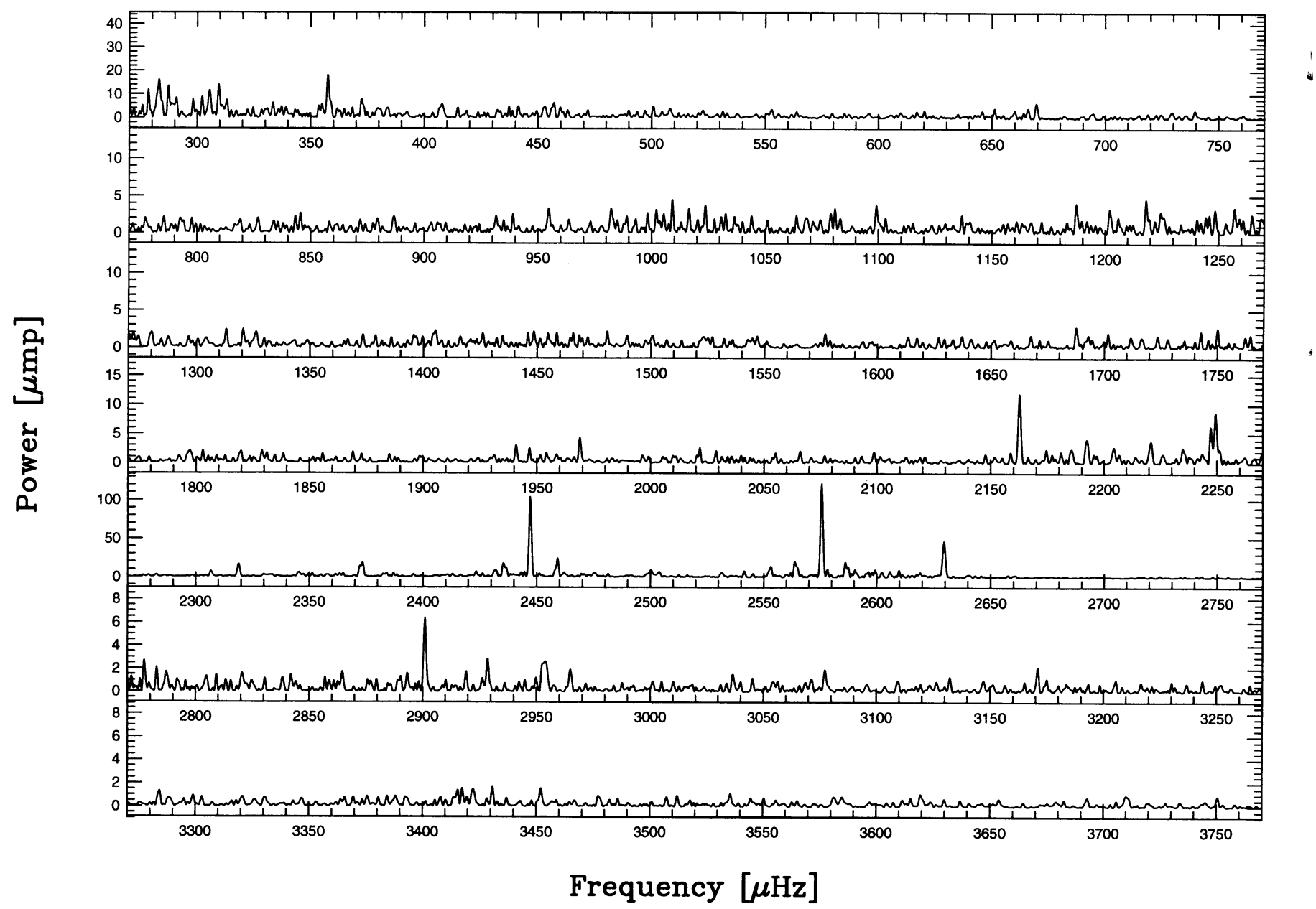

Fig. 3. - Power spectrum of the entire WET run. Note that the vertical scale changes from one panel to the next.

ting these average spectra, we weighted each of the five regions equally, to prevent the high-amplitude peaks from dominating the mean. As indicated in Figure 7, the 1 cycle day ${ }^{-1}(11.6$ $\mu \mathrm{Hz}$ ) alias of the low-frequency peak is present in these spectra. This peak does not represent any real periodicity in the star, though its presence simulates the appearance of quintuplets.

Since these patterns in the spectrum and the averaged spectrum are entirely consistent with the interpretation of their being triplets with approximately equal spacing, we conclude that they are $\ell=1$ mode triplets, with a mean frequency spacing of $27.4 \pm 1.2 \mu \mathrm{Hz}$. While it is possible that these triplets are the result of $\ell=2$ modes with two suppressed components, we show later that the period spacings of the modes confirms their identification as $\ell=1$ modes. This frequency splitting corresponds to one-half the rotation frequency (see eq. [2]), indicating a rotation period of PG 2131 of $5.07 \pm 0.22 \mathrm{hr}$.

In Figures 6 and 7, it is clear that the low-frequency components of the triplets are fully resolved as single, isolated peaks, while the high-frequency peaks are much messier. In the top panel of Figure 7, the large widths of the left and right peaks shows that the splitting is not precisely the same for all modes. These features result from the fact that the highfrequency component is broadened in all cases, and frequently resolved into two or more peaks. The high-frequency component of the multiplet shows fine structure (on the $\mu \mathrm{Hz}$ scale) in all triplets except the one at $384 \mathrm{~s}$. The largest amplitude peak in the fine structure is the highest frequency peak. This fine structure could result from amplitude modulation of a single periodicity, resulting from physical effects such as magnetic and/or nonlinear effects. For the present, we identify the highest amplitude peak within the fine structure as the $m=-1$ peak. In doing so, period spacings between some modes may be affected at the $0.3 \mathrm{~s}$ level because of the inferred $m=0$ positions (see $\S 3.2$ ).

The spacing between the left and right components of the triplets increases slightly with decreasing frequency (i.e., increasing period). Ignoring for the moment the $341 \mathrm{~s}$ mode and considering the next four triplets (periods of 384-450 s), the splitting between the central peak and the dominant highfrequency peak increases steadily from $27.14 \pm 0.15 \mu \mathrm{Hz}$ for the $384 \mathrm{~s}$ peak to $28.51 \pm 0.29 \mu \mathrm{Hz}$ for the $450 \mathrm{~s}$ peak. On the other hand, for the same modes, the frequency splitting of the central and dominant low-frequency peaks alternates between about 26.7 and $28.5 \mu \mathrm{Hz}$. Since the amplitude of this alternation is larger than the trend for the high-frequency components, the total splitting also alternates for consecutive modes in PG 2131.

We show below that there must be a missing triplet between the 341 and $384 \mathrm{~s}$ modes. Given this, the frequency splitting of the $341 \mathrm{~s}$ mode is consistent with the pattern we describe: the missing mode would be a larger than average splitting in the alternating scheme identified above. There is no evidence of 


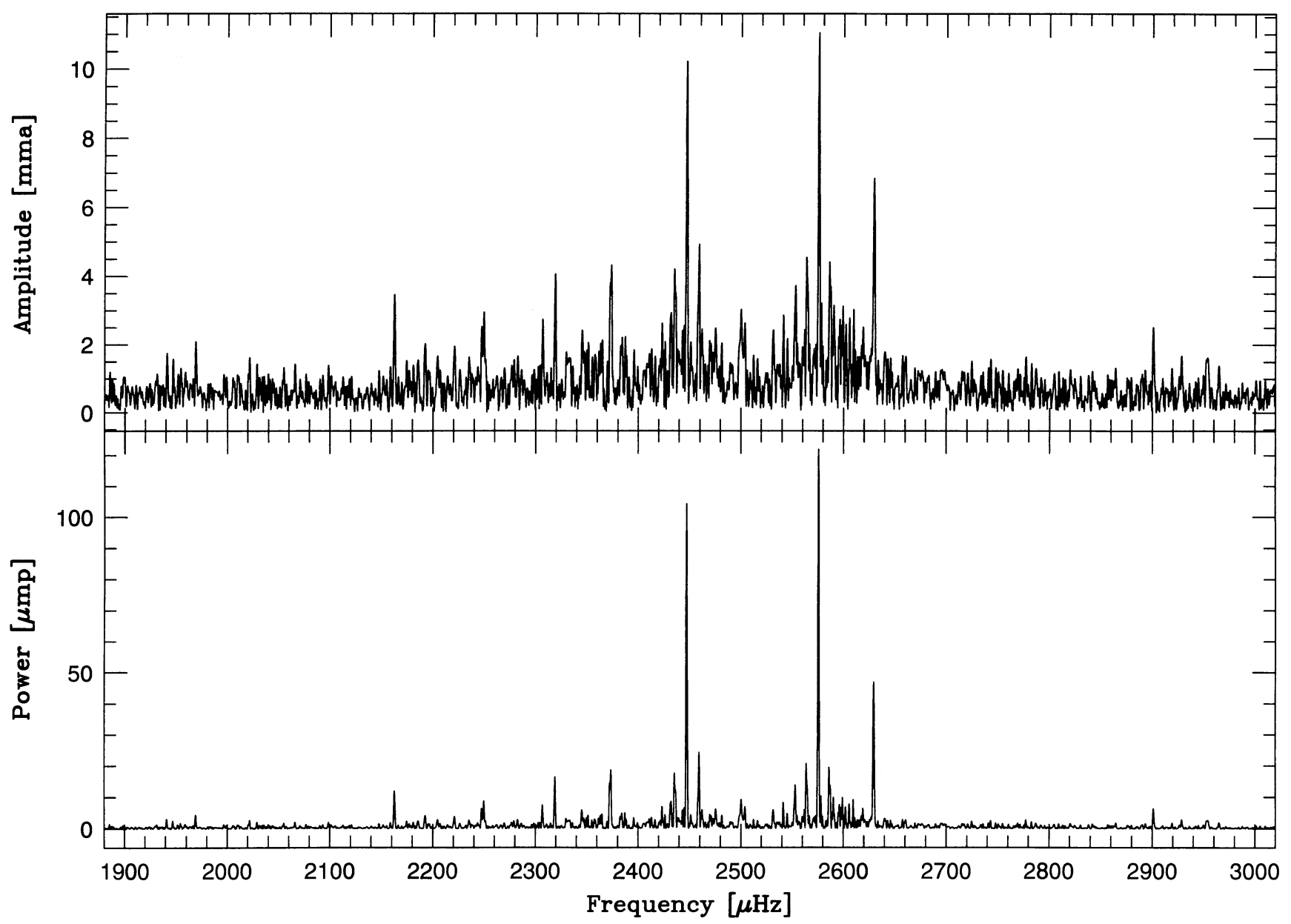

FIG. 4.-Amplitude spectrum (top) and power spectrum (bottom) of the region of largest power in the WET temporal spectrum

pulsations near the expected period in our data. However, in PG 2131 and most pulsating hot white dwarfs, modes are observed to come and go on timescales of months to years. When "new" modes appear, they appear at frequencies that are consistent with pulsation theory; that is, they appear at periods that fit the constant spacing pattern, or at frequencies that match observed rotational splitting values. Therefore, noting that the difference in the splitting between large and small splitting modes (i.e., between the 450.3 and $426.4 \mathrm{~s}$ modes, and the 403.9 and $384.3 \mathrm{~s}$ modes) is about $2.4 \mu \mathrm{Hz}$, we predict that the splitting of the currently missing mode between 341.5 and $384.3 \mathrm{~s}$ will be about $54.7 \mu \mathrm{Hz}$ if and when it is ever observed.

- The relative amplitude of the central peak to the low- and high-frequency peaks in the top panel of Figure 7 is interesting. With this averaging, the two side peaks are at almost equal power, with the central peak at $40 \%$ of the power $(60 \%$ of the amplitude) of the side peaks. Since the triplet structure indicates these are $\ell=1$ modes, the lower amplitude for the $m=0$ mode suggests that we are seeing this star with its rotation axis at high inclination with respect to our line of sight. If the star was viewed from the equator, i.e., high inclination, the amplitude of the $m=0$ mode would be nearly 0 (since the nodal line would then lie at the equator), while if it were pole-on, the

$m=0$ mode would be the largest amplitude, with the $m= \pm 1$ modes showing small amplitudes. Thus, it appears that we are seeing the star mostly equator-on. However, the unequal amplitudes of the $m= \pm 1$ peaks is not easily understood as a purely geometric effect, and must have a deeper explanation.

Another mechanism that can affect the frequency spacing of multiplets is the presence of a magnetic field. Jones et al. (1989) discuss the effects of magnetic fields on pulsating white dwarfs for some simple magnetic field geometries. They show that magnetic fields increase the observed frequencies of modes with all values of $m$. Winget et al. (1994) discuss the combined effects of rotation and magnetic fields in the context of Whole Earth Telescope observations of the DB white dwarf GD 358. For weak magnetic fields in rotating stars, the $\ell=1$ triplets will have the $m=0$ component off-center toward the lower frequency $(m=+1)$ component.

There is no clear asymmetry in the triplet splittings in the WET data for PG 2131. In the case of GD 358, the asymmetry is clear (Winget et al. 1994), but in PG 2131, only two modes show the expected asymmetry, while the other three show either the opposite effect or equal splitting within the uncertainties of the frequencies themselves. From the departures from equality of the splittings, we can derive an upper limit on the magnetic field strength in 1992. Using splittings computed for PG 1159 models by $M$. Montgomery (1994, private communication), a magnetic field of roughly $50,000 \mathrm{G}$ would result in a splitting asymmetry of $1 \mu \mathrm{Hz}$, with the splitting scaling as the square of the magnetic field strength. Therefore 


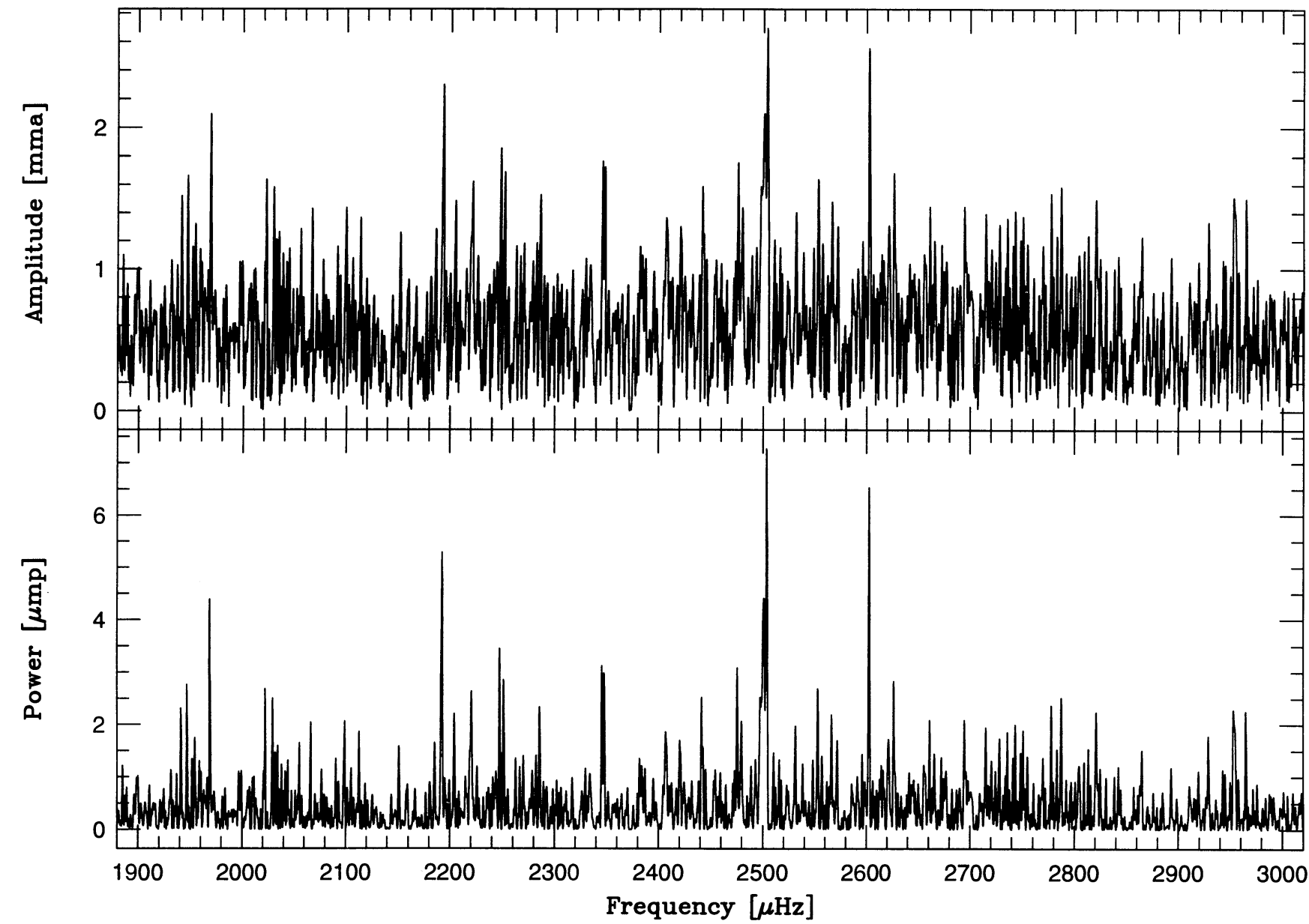

FIG. 5.-Amplitude spectrum (top) and power spectrum (bottom) of the WET data after removal of the nine periodicities labeled "LSQ" in Table 2. These prewhitened spectra show additional peaks.

from the WET data, we can place an upper limit on the magnetic field of PG 2131 of about $50,000 \mathrm{G}$. The upper limit is much lower if the $m=-1$ modes have true frequencies corresponding to the lowest frequency component of the fine structure. Note that this upper limit is much larger than the measurement of the magnetic field of $1300 \pm 300 \mathrm{G}$ for GD 358 (Winget et al. 1994) and the upper limit of $6000 \mathrm{G}$ for PG 1159 (WWET). This is in part a result of the larger uncertainty in the frequency splittings we measure, but is mostly a result of the fact that PG 2131 shows shorter periods than either of those two stars. The splitting by magnetic fields increases rapidly with period (Jones et al. 1989); if PG 2131 showed high-amplitude pulsations at a period of $600 \mathrm{~s}$, then our limit on the magnetic field strength would be similar to that for PG 1159 .

\subsection{Period Spacings}

The oscillations seen in the GW Vir stars are high-overtone nonradial $g$-modes. One of the properties of these modes is that they are approximately equally spaced in period for a given value of $\ell$ and consecutive values of $n$ (see Kawaler \& Bradley 1994, and references therein). The period spacing is determined largely by the mass of the star, with a weak dependence on the stellar luminosity and surface compositional stratification. Therefore, we examined the $m=0$ modes to see if they were nearly equally spaced in period, or separated by integral multiples of the successive period spacing.

Having identified the central $(m=0)$ frequencies for these five triplets, we see that the period spacings are 42.82, 19.66, 22.43, and 23.92 s. Assuming that there is a mode missing between the 341 and $384 \mathrm{~s}$ modes, the mean period spacing is $21.76 \pm 1.57 \mathrm{~s}$. This number is remarkably close to the $21.5 \mathrm{~s}$ mean period spacing for the $\ell=1$ modes in PG 1159-035 reported by WWET. Since the period spacing depends primarily on mass, and only weakly on other stellar parameters, this indicates that PG 2131 has a similar mass to PG 1159 despite its lower luminosity. Ascribing the observed modes to $\ell=2$ gives an unreasonably low mass of $0.39 M_{\odot}$, which is 0.2 $M_{\odot}$ smaller than the mean mass for white dwarfs.

A more rigorous way to determine the period spacing is to fit the periods (and their assumed overtone number) to the form

$$
P_{n}=\Delta P \times\left(n-n_{0}\right)+P_{n_{0}}
$$

to obtain $\Delta P$ and $P_{n 0}$. In the above equation, $\Delta P$ is the mean period spacing, $n$ is the number of radial nodes, and $P_{n_{0}}$ is the fit value of the period for the shortest period mode. A linear least-squares fit of this type yields values of $\Delta P=21.59 \pm 0.37$ $\mathrm{s}$ and $P_{n_{0}}=340.8 \pm 1.2 \mathrm{~s}$. This value of $\Delta P$ is even closer to the value obtained for PG 1159 by WWET than the straight mean. With this information, we could compute an estimated mass 


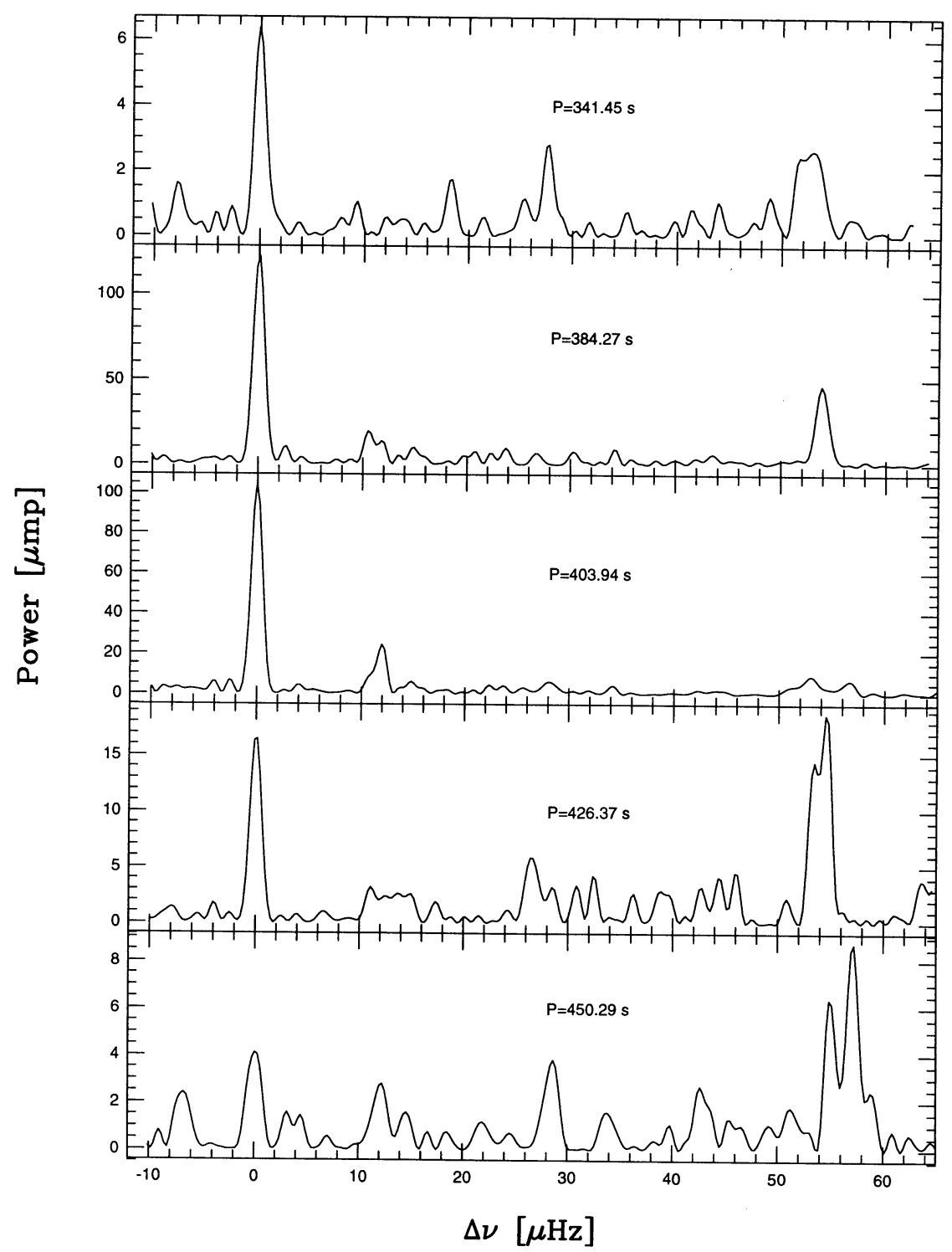

Fig. 6.-Enlargements of the power spectrum for several of the identified triplets. These spectra are aligned on the low-frequency peaks.

for PG 2131, and obtain a model fit to the observed periods. The procedure is described in detail in the next section. Using the best model, we were able to obtain estimates of the overtone number $n$ for each triplet. These are listed in Table 2.

Using the above parameters in equation (3), we estimate the next $m=0$ periods in the sequence to be $470.3(n=19), 491.9$ ' $(n=20)$, and $513.5(n=21)$ s. As we show in the next section, archival data from 1984 shows a peak at a frequency of 2105.9 $\mu \mathrm{Hz}$, which is $56.8 \mu \mathrm{Hz}$ lower than the $2163 \mu \mathrm{Hz}$ peak in the WET data. Thus the $2162 \mu \mathrm{Hz}$ peak corresponds to the highfrequency component of the $n=19$ triplet. While the $m=0$ mode is not seen, the peak seen in 1984 allows us to assume that the $m=0$ mode lies exactly in between the two peaks in frequency, and has a period of $468.54 \mathrm{~s}$. This is a spacing of $18.26 \mathrm{~s}$ from the previous mode. The only other unused mode in the WET data is the $1969 \mu \mathrm{Hz}$ mode, which has no companions. We assume that it is the high-frequency component of the $n=21$ triplet; subtracting the average triplet spacing of 27.4 $\mu \mathrm{Hz}$ gives an estimate of the period of the $m=0$ component of the $n=21$ triplet of $515.08 \mathrm{~s}$. These periods fall reasonably close to the expected values. Assuming these periods are correct, including them in the linear fit as above gives revised values of $21.65 \pm 0.24 \mathrm{~s}$ and $340.5 \pm 1.1 \mathrm{~s}$ for $\Delta P$ and $P_{n_{0}}$, respectively. Their inclusion into the fit does not change the values of the fit parameters significantly.

With these seven periods, we can apply some statistical tests to demonstrate the level of confidence with which we can believe the equal period spacing model. The first test is the K-S test (Kawaler 1988). The results of this test are shown in Figure $8 a$. The deep and choppy minimum between 20 and $25 \mathrm{~s}$ suggests that the spacing we have identified is significant, but that variations away from the mean spacing are relatively large. Comparison with the same test using the pre-WET periods of PG 1159 confirms this suspicion. Figure $8 b$ shows a new test for period spacings proposed by O'Donoghue (1994). This method computes the inverse of the variance of the residuals of the fit above as a function of period spacing. This plot shows a single large peak at the same period spacing as the linear fit 

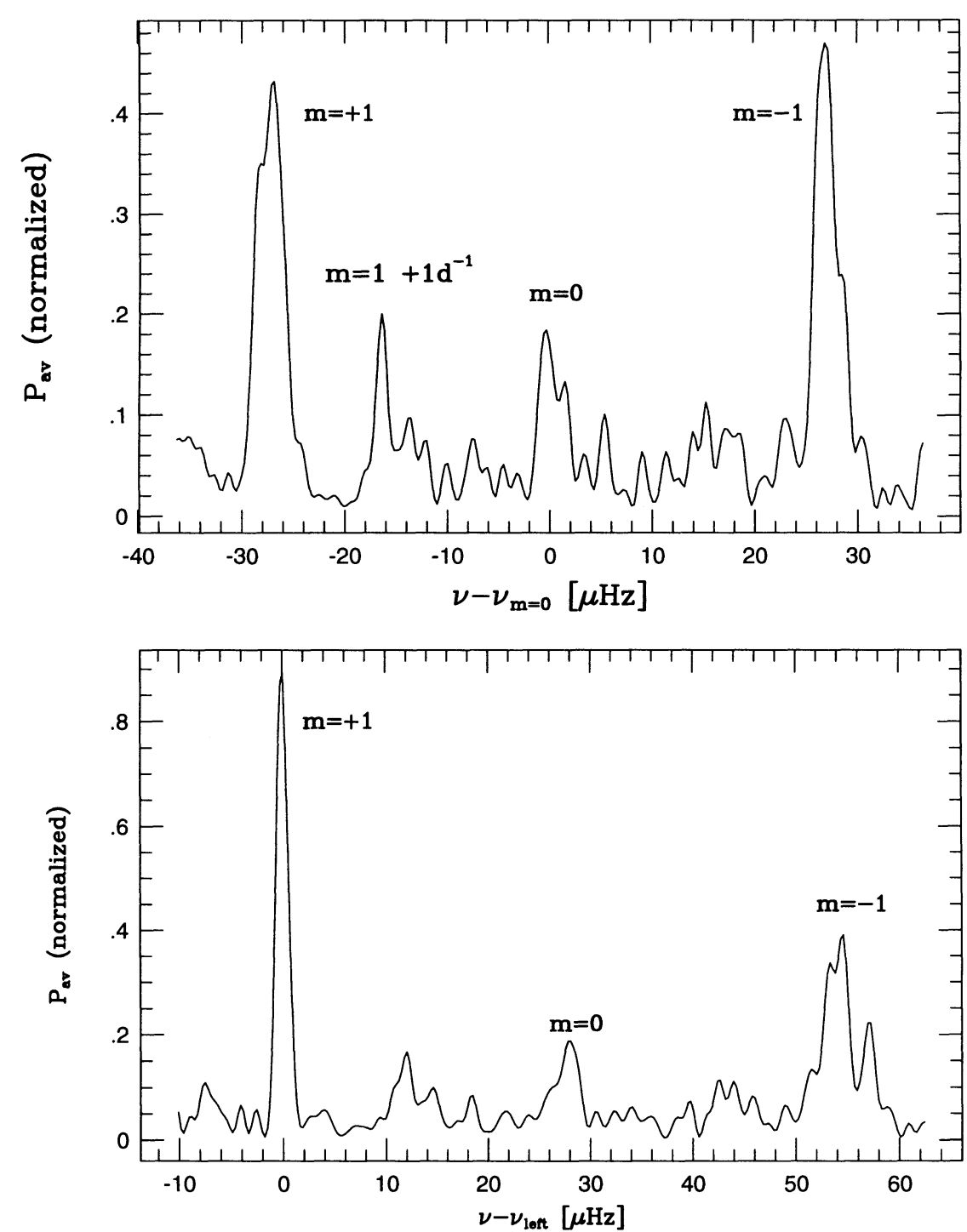

FIG. 7.-Averaged triplet shape. In both panels, each triplet was weighted equally to prevent the large-amplitude modes from dominating the average. Top: triplets were aligned on the $m=0$ position. Bottom: triplets aligned by the low-frequency component.

above. Thus we have confidence in the identified period spacing of $21.65 \pm 0.24 \mathrm{~s}$.

We note that the spread in the measured period spacings is much larger than the uncertainty in the periods themselves, or in the estimate of $\Delta P$. This reflects the effects of mode trapping on period spacings, as described in detail in Kawaler \& Bradley (1994). Figure 9 shows the departures from uniform period spacing for these modes. The top panel plots the difference between the observed period and the periods calculated with equation (2), using a mean period spacing of $21.64 \mathrm{~s}$, while the bottom panel plots the difference between the period and the next lowest period. In cases where a period is missing from the sequence we plot an open circle at the position of the affected period in the bottom panel. Figure 9 shows that the departures from uniformity show a repeating pattern, with a cycle of about three modes. The magnitude of the departures is 2-3 s. The models of Kawaler \& Bradley (1994) are consistent with this behavior when the helium layer is thicker than 6.2 $\times 10^{-3} M_{*}$, and the surface helium abundance is $0.3-0.5$ by mass (see their Figs. 7 and 9).
The relatively small number of modes in PG 2131 prevents us from being nearly as precise as was possible with PG 1159 . A better signal-to-noise ratio for this run might have uncovered more modes, but the small number of telescopes, combined with the faintness of the star and the small average apertures of the telescopes did not allow these modes to peek above the noise. We did look for lower amplitude modes by looking at subsets of the data of higher signal-to-noise, but these searches were inconclusive. We also looked at short segments of the run to find short-lived modes, but this too was inconclusive. It did show that the $m=-1$ modes have unstable amplitudes, as expected given their fine splitting, but no other modes were found.

\subsection{The Mass, Luminosity, and Distance to PG 2131}

We now use the observed period spacings to estimate the global parameters of PG 2131 using the technique described in Kawaler \& Bradley (1994). First we estimate the mass using the observed mean period spacing. The gravity and effective temperature quoted by Dreizler et al. (1995) using optical spectra 


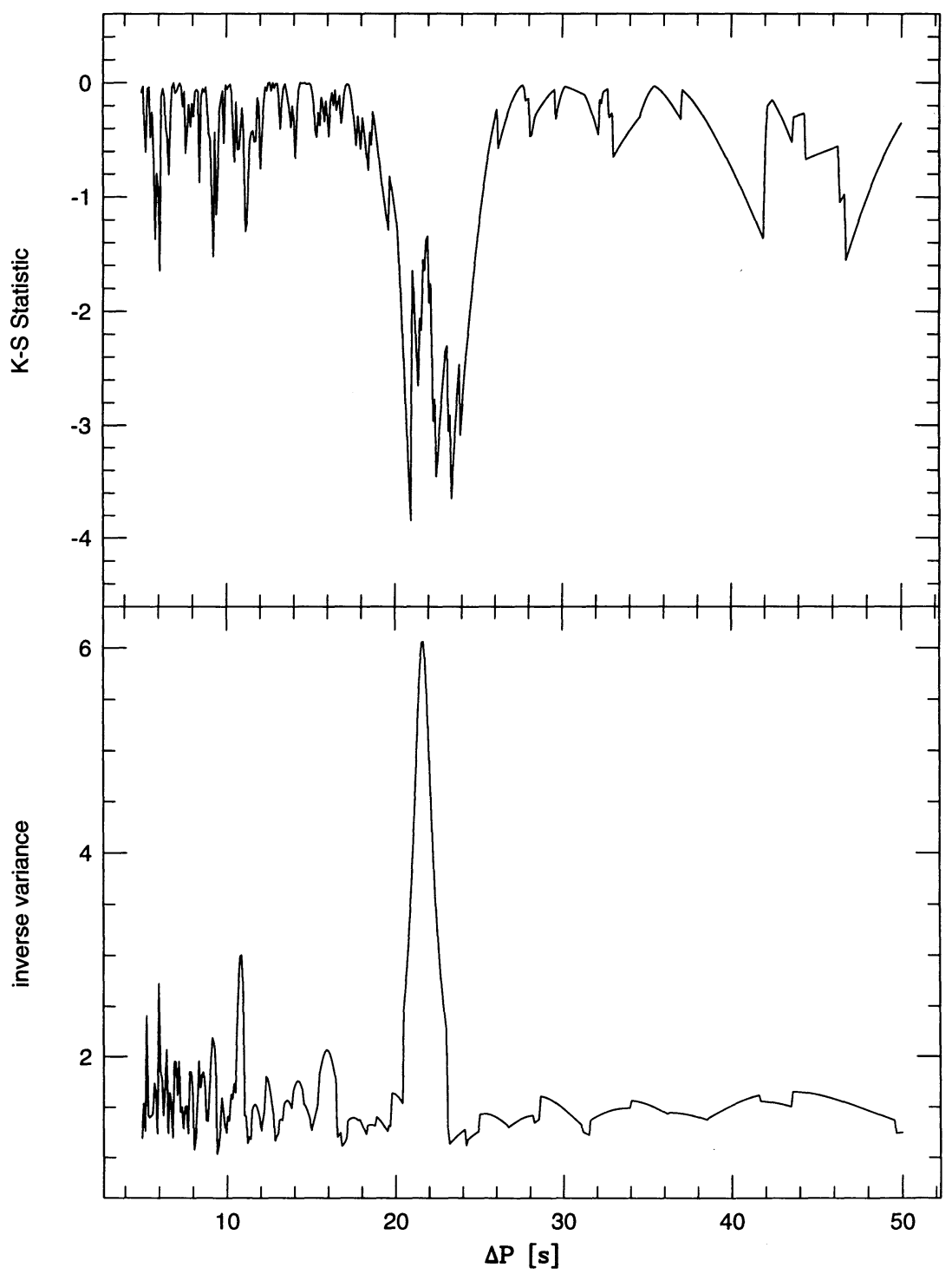

Fig. 8.-Two significance tests for uniform period spacings. In both tests, the best estimates for eight $m=0$ frequencies were used. Top: Kolmogorov-Smirnov test. Bottom: inverse variance test from O’Donoghue (1994). Both test show the significant period spacing at $21.6 \mathrm{~s}$.

suggest $\log \left(L / L_{\odot}\right)=1.3 \pm 0.5$. This is ten times smaller than found for PG 1159. Since models with lower luminosity must have higher mass to maintain the observed period spacing, they imply that PG 2131 has a higher mass than PG 1159 if the Dreizler et al. (1995) temperature and gravity estimates are correct. Their luminosity estimate places PG 2131 at a lumi' nosity that is lower than the models used in Kawaler \& Bradley (1994), but extrapolating their equation (5) provides an initial mass estimate of $0.62 \mathrm{M}_{\odot}$.

We computed some preliminary stellar models of PG 2131 to determine the stellar properties using a new stellar evolution code (ISUEVO) designed for evolutionary and pulsational studies of white dwarf and pre-white dwarf stars. The input physics for these models is similar to that used in the static models described in part in Kawaler \& Bradley (1994); further details about ISUEVO can be found in Dehner \& Kawaler (1995). With the above mass estimate as a starting point, we computed evolutionary models with masses from 0.58 to 0.62 $M_{\odot}$ from higher luminosity than PG 1159 to lower luminosity than the Dreizler et al. (1995) estimate for PG 2131. The compositional stratification in all of these preliminary models is the same as the best model of PG 1159 in Kawaler \& Bradley (1994). We stress that the results presented here are preliminary in that we have not tried to fit the details of the period distribution, but only to match the observed $m=0$ periods to within 1 or $2 \mathrm{~s}$.

The general behavior of the mean period spacings follows the description in Kawaler \& Bradley (1994). We can fit the observed periods very precisely with reasonable model parameters. Unlike PG 1159, there is not a unique fit for PG 2131. For an evolutionary sequence of a given mass, the model periods fit the observed periods at two different temperatures. A high-temperature model that shows a close fit to the observed periods also shows periods that are (generally) increasing with time. Some time later, at a lower temperature, the accumulated secular period increase equals the spacing between modes of consecutive $n$, so that the model fits the star again. At that lower luminosity, the fit is as close as at the 


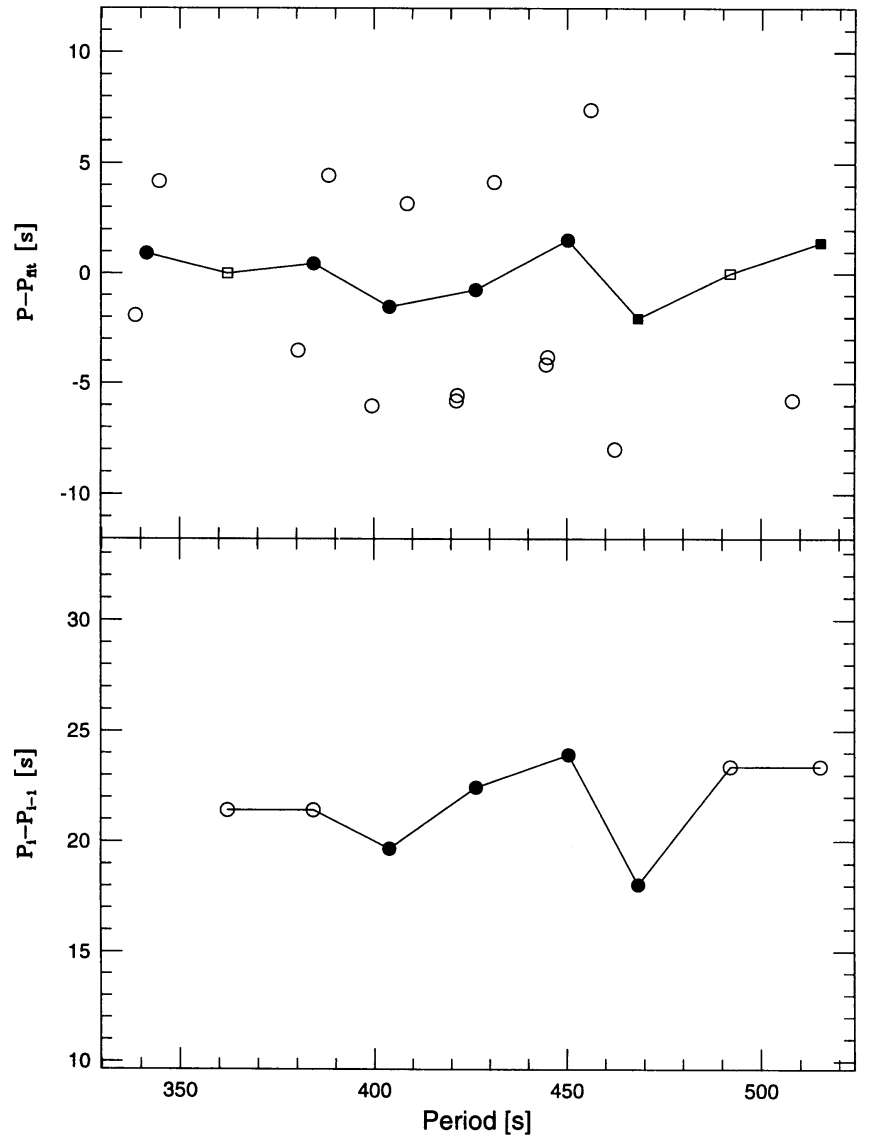

Fig. 9.-Period spacings in PG 2131 are represented in two ways. Top: difference between the observed period and the period obtained by the linear fit in eq. (1), assuming a period spacing of $21.64 \mathrm{~s}$. Bottom: period difference (as a function of period). In both cases, open circles correspond to non $m=0$ peaks, and solid circles correspond to periods of $m=0$ modes. The open squares in the top panel represent "missing" modes.

higher luminosity. Because only one trapping cycle is clear in the period spacing diagram, there is no purely seismological constraint on the effective temperature (or, equivalently, the luminosity) so we cannot choose between the two models without additional constraints.

If we accept the spectroscopic constraints of Dreizler et al. (1995), then we can choose between the two models. In our 0.62 $M_{\odot}$ sequence, the models that fit the periods seen in PG 2131 have effective temperatures of 97,100 and $80,600 \mathrm{~K}$. Our $0.60 M_{\odot}$ sequences fit PG 2131 at effective temperatures of 114,000 and $88,100 \mathrm{~K}$. The Dreizler et al. (1995) effective temperature of PG $2131,80,000 \pm 10,000 \mathrm{~K}$, eliminates the hightemperature solution at the $2 \sigma$ level, except for masses above about $0.65 M_{\odot}$. The values of $n$ listed in Table 2 are from the lower temperature models. With this constraint, our preliminary estimate of the mass of PG 2131 is $0.61 \pm 0.02 M_{\odot}$, and $\log \left(L / L_{\odot}\right)=1.0 \pm 0.2$. The luminosity estimate is based on the luminosities corresponding to the best models for each mass that also satisfy the $T_{\text {eff }}$ constraint. With a $V$-magnitude of 16.6 , and assuming a bolometric correction of approximately $6.0 \pm 0.5$ (extrapolating results of WHH for hotter stars) results in a distance of $470_{-130}^{+180} \mathrm{pc}$, where the quoted errors are dominated by the uncertainty in the bolometric correction and in the luminosity.

Though this distance is a factor of 2 smaller than the esti- mate by Wesemael et al. (1985), it is only a little more than $1 \sigma$ below their estimate of $1047_{-500}^{+1000} \mathrm{pc}$. At a Galactic latitude of $-31^{\circ}$, their estimate places PG 2131 over $500 \mathrm{pc}$ out of the plane of our Galaxy. With our distance to PG 2131 of $470 \mathrm{pc}$, we return it to the comfort of the galactic plane- $240 \mathrm{pc}$ below the plane, and within the scale height of white dwarfs and planetary nebulae. A distance estimate using the HSTmeasured brightness of the companion will provide a useful check of the distance to PG 2131.

\section{EIGHT YEARS BEFORE WET: ARCHIVAL DATA FOR PG 2131}

In this section, we describe results of rereduction of archival data on PG 2131 obtained at McDonald Observatory in 1984. This independent data set showed several periodicities, and with the 20/20 hindsight of the current WET run, allows several interesting comparisons. Soon after the discovery of the variability of PG 2131 by Bond et al. (1984), J. Allen Hill and R. E. Nather observed this star with the UT 2 star photometer on the 82 inch telescope at McDonald Observatory. The data were obtained on five nights (spread over 7 days) in 1984 September. The logs for these runs are shown in Table 3, and show that the overall duration of the runs was 6.06 days, with data obtained on five of the seven available nights. The raw data, obtained from the archives of the photometry group at the University of Texas, were reduced and analyzed for this study using the same techniques as for the WET data.

In identifying peaks in this data set, we worked independently from the WET set as much as possible. We performed successive least-squares fits to the data, beginning with the largest amplitude peaks and working down in amplitude. At each stage, we increased the number of modes included in the fit. To aid in selecting the next peak for a fit, we computed and Fourier transformed prewhitened data sets. At many points in the analysis the 1 cycle day ${ }^{-1}$ and $\frac{1}{6}$ cycle day $^{-1}$ aliases prevented an unambiguous selection of the next peak to fit using only the 1984 data. In these circumstances, we used the WET results to determine which peaks were in the star and which were aliases.

We still are impressed by the impossibility of uniquely identifying true signals in the data without the Whole Earth Telescope. The upper panel of Figure 10 shows the power spectrum of the 1984 data, while the bottom panel of Figure 10 shows the power spectrum after prewhitening by the four largest peaks, after those peaks were identified with reference to the WET data. In the upper panel, the peak near $2250 \mu \mathrm{Hz}$ shows the largest peak at the -1 cycle day $^{-1}$ alias of the true peaks. In the lower panel, the positions of the true peaks are indicated with arrows; in most cases this data set shows alias peaks higher than the true peaks. The reason for this is twofold. First, the data gaps resulted in a complicated window pattern domi-

TABLE 3

ObServing Log for the ARChive McDonald 82 INCH OBSERVATIONS OF PG $2131+066$

\begin{tabular}{lccc}
\hline \hline Run Name & $\begin{array}{c}\text { Start Date } \\
\text { (UT) }\end{array}$ & $\begin{array}{c}\text { Start Time } \\
\text { (UT) }\end{array}$ & $\begin{array}{c}\text { Duration } \\
\text { (h:m:s) }\end{array}$ \\
\hline r2990 ..... & 1984 Sep 18 & $02: 55: 00$ & $5: 48: 50$ \\
r2992 ..... & 1984 Sep 19 & $02: 22: 00$ & $5: 20: 00$ \\
r2994 ..... & 1984 Sep 22 & $02: 31: 01$ & $3: 09: 20$ \\
r2995 ..... & 1984 Sep 23 & $02: 10: 01$ & $4: 41: 40$ \\
r2996 ..... & 1984 Sep 24 & $02: 12: 50$ & $2: 21: 50$ \\
\hline
\end{tabular}




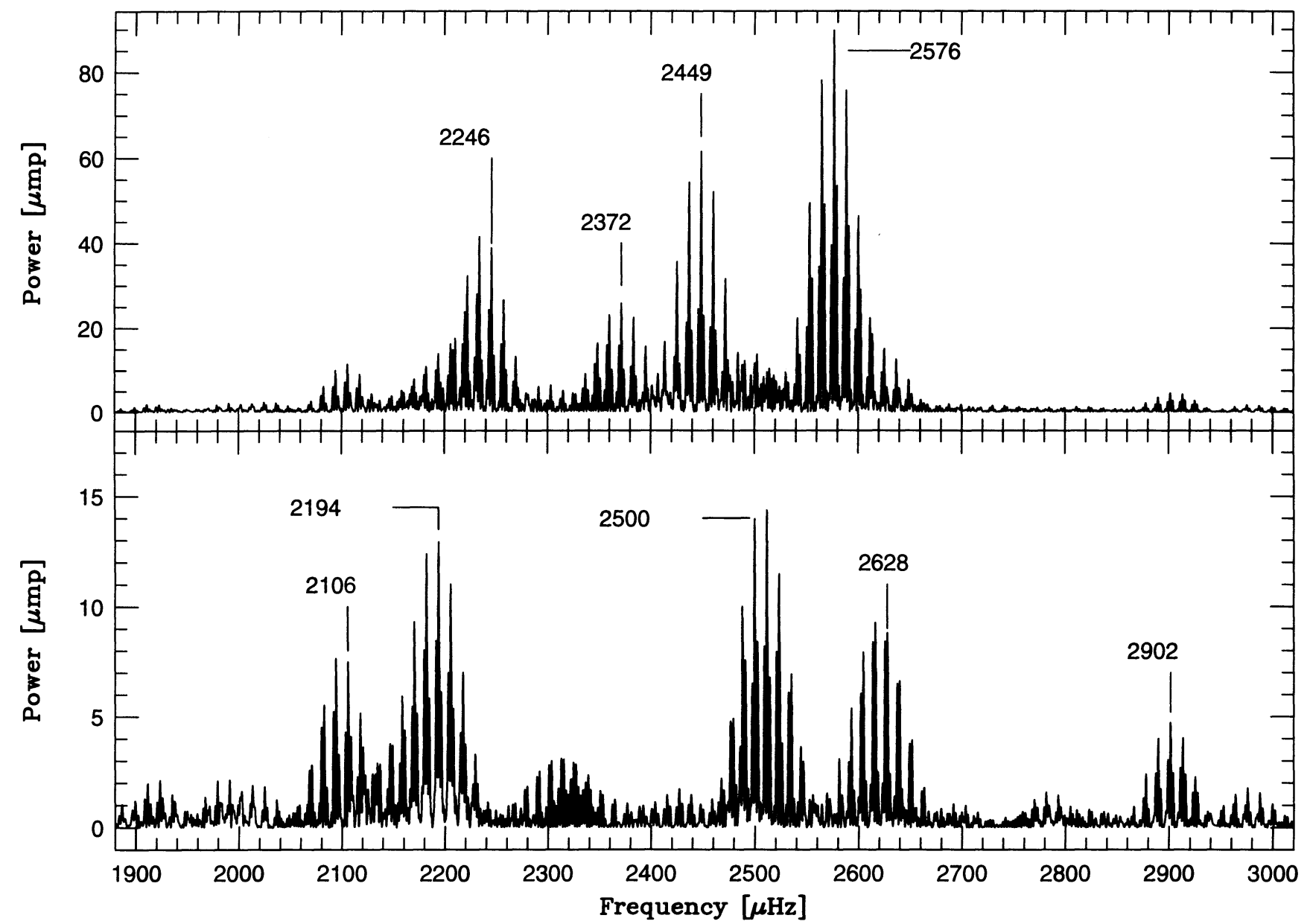

FIG. 10.-Power spectrum of the combined archival data on PG 2131 obtained at McDonald Observatory in 1984. The top panel shows the power spectrum. The bottom panel shows the power spectrum of the data after the four largest amplitude periodicities were removed (in the time domain). The positions of the peaks closest to those in our data are indicated.

nated by 1 cycle day ${ }^{-1}$ and $\frac{1}{6}$ cycle day ${ }^{-1}$ aliases. Second, PG 2131 itself shows frequency spacings near 5 cycles day $^{-1}$ in individual triplets, and overlaps between triplets are frequently small-integral multiplets apart (i.e., 6 cycles day ${ }^{-1}$ between the $2391 \mu \mathrm{Hz}$ and $2249 \mu \mathrm{Hz}$ peaks, etc.). Thus, these data are a mess that requires global observations to sort out. Though imperfect, this WET run was sufficient to solve almost all of the alias problems. While the frequencies are accurate, the amplitude of theses modes are suspect. Even with least-squares fitting, noise effects do not allow us to determine with confidence the true amplitude of peaks whose window functions overlap.

- We eventually found eight peaks in the 1984 data. Seven of the eight peaks have frequencies very close to where peaks were found in the WET data. The results of the analysis are shown in Table 4. The eighth peak in the 1984 data fell at $2105.9 \mu \mathrm{Hz}$, where no power is present in the WET data. However, this peak lies $56.1 \mu \mathrm{Hz}$ lower in frequency than the $2162.68 \mu \mathrm{Hz}$ peak in the WET data. Therefore, we identify the mode seen in 1984 as the left-hand peak corresponding to the $2162.68 \mu \mathrm{Hz}$ peak in the WET data. This confirms our previous contention that the period of the $m=0$ mode in that triplet lies to the low-frequency side of the $2162.68 \mu \mathrm{Hz}$ peak. We note that we found no evidence for $m=0$ modes in the 1984 data. Given their low amplitudes in the WET data, the 1 cycle day ${ }^{-1}$ alias problem could easily have hidden evidence for $m=0$ modes. Therefore, the fact that we were unable to detect them in the archival data does not mean that they were not there. Even if they were not, it would not be unusual, based on experience with other stars.

The final column of Table 4 shows the difference in frequency in the identified modes from the 1992 WET run and the 1984 run. All of the peaks that we found in 1984 are signifi-

TABLE 4

Periodicities of PG $2131+066$ Observed in 1984 SePtember (HILL)

\begin{tabular}{ccccc}
\hline \hline $\begin{array}{c}\text { Frequency } \\
(\mu \mathrm{Hz})\end{array}$ & $\begin{array}{c}\text { Amplitude } \\
(\mathrm{mma})\end{array}$ & $m$ & $n$ & $\begin{array}{c}\Delta f, 1992-1984 \\
(\mu \mathrm{Hz})\end{array}$ \\
\hline $2901.55 \pm 0.12 \ldots$ & $2.16 \pm 0.31$ & 1 & 13 & $-0.58 \pm 0.20$ \\
& & & & \\
$2627.97 \pm 0.08 \ldots$ & $3.30 \pm 0.31$ & -1 & 15 & $+1.50 \pm 0.10$ \\
$2576.45 \pm 0.03 \ldots$ & $9.57 \pm 0.31$ & 1 & & $-0.91 \pm 0.05$ \\
$2500.04 \pm 0.07 \ldots$ & $3.97 \pm 0.31$ & -1 & 16 & $+3.46 \pm 0.19$ \\
$2448.76 \pm 0.04 \ldots$ & $6.37 \pm 0.31$ & 1 & & $-1.55 \pm 0.06$ \\
$2371.61 \pm 0.05 \ldots$ & $4.92 \pm 0.31$ & -1 & 17 & $+1.94 \pm 0.14$ \\
$2245.64 \pm 0.05 \ldots$ & $5.62 \pm 0.31$ & -1 & 18 & $+3.69 \pm 0.14$ \\
$2193.94 \pm 0.07 \ldots$ & $3.83 \pm 0.31$ & 1 & & $-1.61 \pm 0.17$ \\
$2105.90 \pm 0.09 \ldots$ & $2.94 \pm 0.31$ & -1 & 19 & $\ldots$ \\
\hline
\end{tabular}


cantly different from their 1992 counterparts. Those peaks that have $m=+1$ are all at lower frequency in 1992 than in 1984, while the $m=-1$ peaks are all at higher frequencies. Because of the fine structure seen in the $m=-1$ peaks (see Figs. 6 and 7 ), we should qualify this statement. In Table 4, the frequency changes in the $m=-1$ modes are given for the largest amplitude peak at the $m=-1$ position in 1992. In two cases, the $m=-1$ peak in 1984 corresponds very closely to a peak within the $m=-1$ fine structure in the 1992 data. Therefore, it is not clear whether the frequency changes in these $m=-1$ modes are indeed as large as indicated in this column. It is possible that a different component of the fine structure in these $m=-1$ modes was at a larger amplitude in 1984 than in 1992.

These observed frequency changes are much larger than we expect if they are caused by secular (evolutionary) effects. To put things in perspective, the $2447 \mu \mathrm{Hz}$ mode decreased in frequency by $1.55 \pm 0.06 \mu \mathrm{Hz}$ in 8 years. If this change occurred at a constant rate, then the rate of period change is $d P / d t=(1.02 \pm 0.04) \times 10^{-9} \mathrm{~s} \mathrm{~s}^{-1}$. Converting this to a timescale for period change gives $\tau_{P}=1.5 \times 10^{4} \mathrm{yr}$. This apparent rate of period change in PG 2131 is almost 2 orders of magnitude faster than for PG 1159 (WWET). Since PG 2131 has a lower luminosity and temperature than PG 1159 , it is probably an older object that is evolving more slowly; the frequency changes are probably caused by something other than the secular evolution of PG 2131.

All of the $m=+1$ modes have frequencies that decreased between 1984 and 1992, while all of the $m=-1$ modes have frequencies that increased in the same time interval. Thus the total splitting of the triplets increased by approximately 2 to 4 $\mu \mathrm{Hz}$ between 1984 and 1992. If we attribute the splitting entirely to rotation, the frequency changes could result from an increase in the rotation rate of PG 2131 by about $3 \mu \mathrm{Hz}$ in 8 years. This implies a rate of increase of the rotation frequency of $1.2 \times 10^{-14} \mathrm{~s}^{-2}$. Given the rotation period itself is a bit over $5 \mathrm{hr}$, this corresponds to a spin-up timescale of 145 years. Spin up resulting from conservation of angular momentum in a star that is contracting, and therefore decreasing its moment of inertia, must occur on the timescale of contraction. The timescale for contraction is much longer than 100 years in white dwarf models. Thus simple rotational spin-up is unlikely to be the cause of the observed frequency changes in PG 2131.

Because of the complexity of the power spectrum around most of the $m=-1$ peaks and the very large apparent frequency changes it is possible that these two related phenomena are caused by nonlinear effects. The three modes in each triplet are nearly in 1:1 resonance, and so mode-mode coupling can be responsible for much of the complexity. Such coupling naturally explains why the amplitudes of the $m=+1$ and $m=-1$ modes differ within a triplet. Buchler, Goupil, \& Serre (1995) have studied the consequences of such a coupling; their work may be extremely relevant for PG 2131.

\section{SUMMARY AND CONCLUSIONS}

Temporal spectroscopy of PG 2131 with the Whole Earth Telescope revealed a number of details about this star. It pulsates in $\ell=1$ modes with several consecutive overtones present. This star rotates with a period of $5.07 \mathrm{hr}$. It has a mass of $0.61 \pm 0.02 M_{\odot}$, and there is a discontinuity in the composition below about $6 \times 10^{-3} M_{*}$ below the surface. It lies at a distance of $470 \pm 150 \mathrm{pc}$. The surface helium abundance is approximately $0 . \overline{3}$ to 0.5 by mass. The overall frequency splitting shows alternating values about an increasing mean, suggesting either the effects of mode trapping or of magnetic fields. The global magnetic field is probably less than 50,000 G. Data taken 8 years earlier indicate that the width of several triplets has increased by up to $10 \%$. The frequency changes are unlikely to have been caused by secular effects or by rotation. The distribution of power within triplets, the complex structure in the power spectrum surrounding the prograde mode, and the observed frequency changes all point toward nonlinear mode coupling influencing the observed frequencies. Clearly, more detailed calculations with appropriate models will be able to address this question.

PG 2131 shows some similarities to PG 1159, but also some important differences. The largest amplitude periods are at higher frequency than PG 1159. There are fewer periods present, however, and there is no evidence for $\ell=2$ modes. The amplitude of the pulsations is slightly larger in PG 2131 than in PG 1159. The differences in the pulsation behavior are probably correlated to the difference in temperature between PG 1159 and PG 2131, as the trends are obeyed by other pulsators of this class. Understanding these correlations is a high-priority goal of future Whole Earth Telescope observations along with theoretical investigations of this important class of stars.

We thank a host of funding agencies from around the world. In particular, S. D. K. acknowledges the National Science Foundation for support under the NSF Young Investigator Program (grant AST 92-57049) and grant AST 91-15213 to Iowa State University. J. C. C. enjoys support as a Hubble Fellow, through NASA grant number HF-1041.01-93A from the Space Telescope Science Institute, which is operated by the Association of Universities for Research in Astronomy, Inc., under NASA contract NAS 5-26555. The Polish WET group is supported by the Polish Committee for Scientific Research through grant no. 2-2109-91-02. The Texas WET group acknowledges support from NSF grant AST 90-14655 to the University of Texas. MAW received partial support through NSF grant AST 92-17988 to Florida Institute of Technology. Communication between the WET operation headquarters at the University of Texas and observers at Mount Maidanak was with the assistance of the Technical University of Berlin, Institut für Luft- und Raumfart, through the TURSAT satellite.
Bond, H. E., Grauer, A. D., Green, R. F., \& Liebert, J. 1984, ApJ, 279, 751 Brickhill, A. J. 1975, MNRAS, 170, 404

Buchler, J. R., Goupil, M. J., \& Serre, T. 1995, A\&A, in press

Cox, J. P. 1980, Stellar Pulsation (Princeton: Princeton Univ. Press) Dehner, B. T., \& Kawaler, S. D. 1995, ApJ, in press

Dreizler, S., et al. 1995, in 9th European Workshop on White Dwarf Stars, ed. D. Koester \& K. Werner (Dordrecht: Kluwer), in press

Hill, J. A. 1986, M.S. thesis, Univ. Texas

Jackson, J. D. 1975, Classical Electrodynamics (2d ed.; New York: Wiley)

Jones, P. W., Pesnell, W. D., Hansen, C. J., \& Kawaler, S. D. 1989, ApJ, 336, 403

Kawaler, S. D. 1988, in Advances of Helio- and Asteroseismology, ed. J. Christensen-Dalsgaard \& S. Frandsen (Dordrecht: Reidel), 329

\section{REFERENCES}

Kawaler, S. D., \& Bradley, P. A. 1994, ApJ, 427, 415

Nather, R. E., Winget, D. E., Clemens, J. C., Hansen, C. J., \& Hine, B. P. 1990 , ApJ, 361, 309

O'Donoghue, D. 1994, MNRAS, 270, 222

Unno, W., Osaki, Y., Ando, H., Saio, H., \& Shibahashi, H. 1989, Nonradial Oscillations of Stars (2d ed.; Tokyo: Univ. Tokyo Press)

Werner, K., Heber, U., \& Hunger, K. 1991, A\&A, 244, 437

Wesemael, F. Green, R. F., \& Liebert, J. 1985, ApJS, 58, 379

Winget, D. E., et al. 1991, ApJ, 378, 326 (WWET)

. 1994, ApJ, 430, 839 